\title{
Optically Controlled Energy Transfer in Stacked and Co-Planar Polycyclic Chromophores
}

\author{
David S. Bradshaw, Jamie N. T. Peck, Vasily S. Oganesyan* and David L. Andrews* \\ School of Chemistry, University of East Anglia, Norwich NR4 7TJ, United Kingdom \\ *To whom correspondence should be addressed. E-mail: d.1.andrews@uea.ac.uk; \\ v.oganesyan@uea.ac.uk
}

\section{RECEIVED DATE}

In the search for enhanced control over the process of resonance energy transfer in multichromophore molecular systems, all-optical mechanisms offer many significant advantages over other systems. One recently conceived scheme, based on the optical switching of energy transfer, is achieved by coupling a normally forbidden decay transition with pulses of off-resonant laser light. Earlier work has suggested that such systems could offer levels of efficiency that might approach those associated with the usual Förster mechanism. In this Letter, the ab initio results of specific calculations on stacked and co-planar polycyclic chromophores are reported. The results show that by judicious choice of electronic state and laser wavelength, much higher levels of efficiency are achievable. A possible scheme for the implementation of such a system is discussed with regard to its potential use in energy harvesting and optical switching applications. 


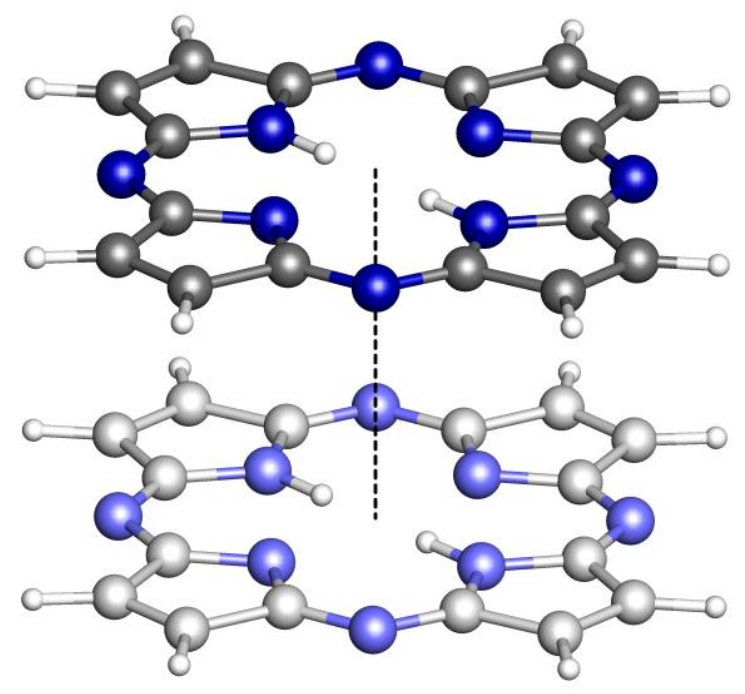

KEYWORDS: Energy transfer, nonlinear optics, computational chemistry, quantum theory, ab initio calculations

Quantum electrodynamics (QED) is the analytical tool of choice for the theory of optical processes involving molecular systems. ${ }^{1-3}$ Although the basic mechanisms of photophysics and photochemistry (e.g. single-photon absorption) are easily described by QED, the main focus in recent years has been the discovery and characterization of novel nonlinear processes yet to be explored by experimentation. Over several decades, and before recent advances in computational chemistry, the capacity to apply QED to specific, optically nonlinear materials has been constrained by the difficulty of evaluating the necessary properties of higher energy electronic states. With quantitative data on key optical parameters now being computationally accessible, this Letter represents a report of the very first application of QED to an optical process in pre-defined molecular systems. In detail, computational calculations are here used to secure $a b$ initio numerical results quantifying the propensity of a purposely designed system, comprised of polycyclic chromophores (specifically porphine) arranged in a stacked or co-planar configuration (Fig. 1), to exhibit optically controlled energy transfer. 


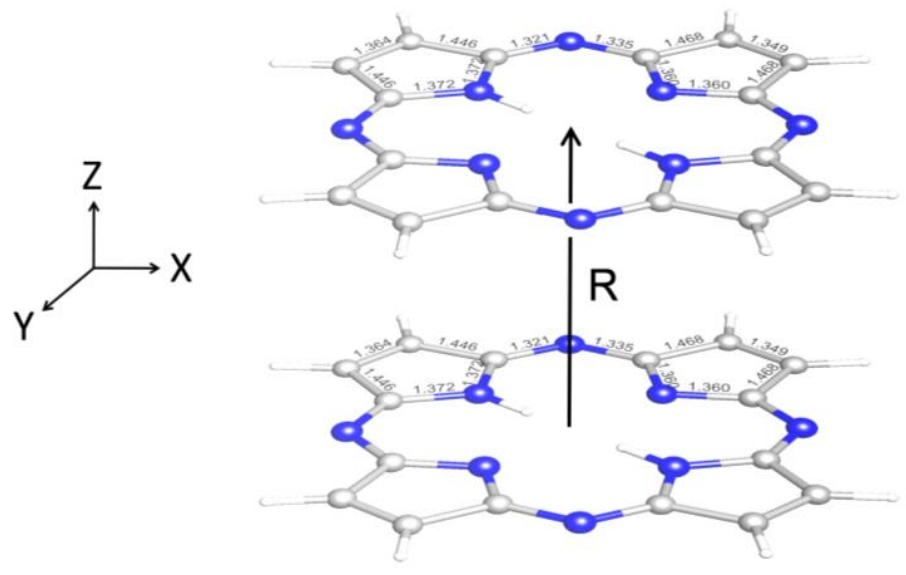

(a)
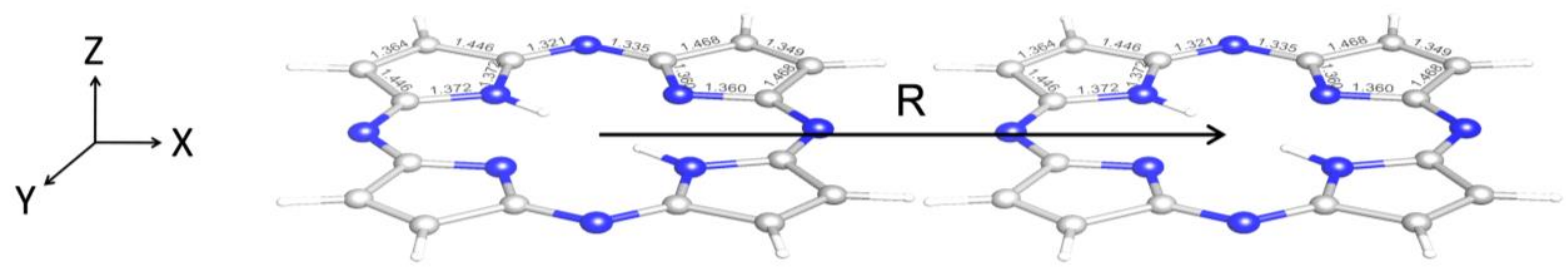

(b)

Fig. 1. Illustration of porphine $\left(\mathrm{C}_{20} \mathrm{H}_{14} \mathrm{~N}_{4}\right)$ chromophores arranged in $(a)$ stacked and $(b)$ co-planar configurations, which correspond to a displacement vector, $\mathbf{R}$, directed in the $\mathrm{Z}$ and $\mathrm{X}$ directions, respectively.

In any such multi-chromophore system, the absorption of visible or ultraviolet light generally leads to the acquisition of energy by constituent chromophores. Within the system, this electronic energy will often transfer from one chromophore to another by a familiar mechanism known as Förster transfer. ${ }^{4-6}$ In a recent report, ${ }^{7}$ a novel aspect of this mechanism has been identified, by means of which off-resonant laser pulses can facilitate energy transfer that could not otherwise take place; this defines the concept of optically controlled resonance energy transfer (OCRET), a conceivable basis for all-optical switching. With the adoption of anticipated, order of magnitude values for salient parameters, anticipated transfer 


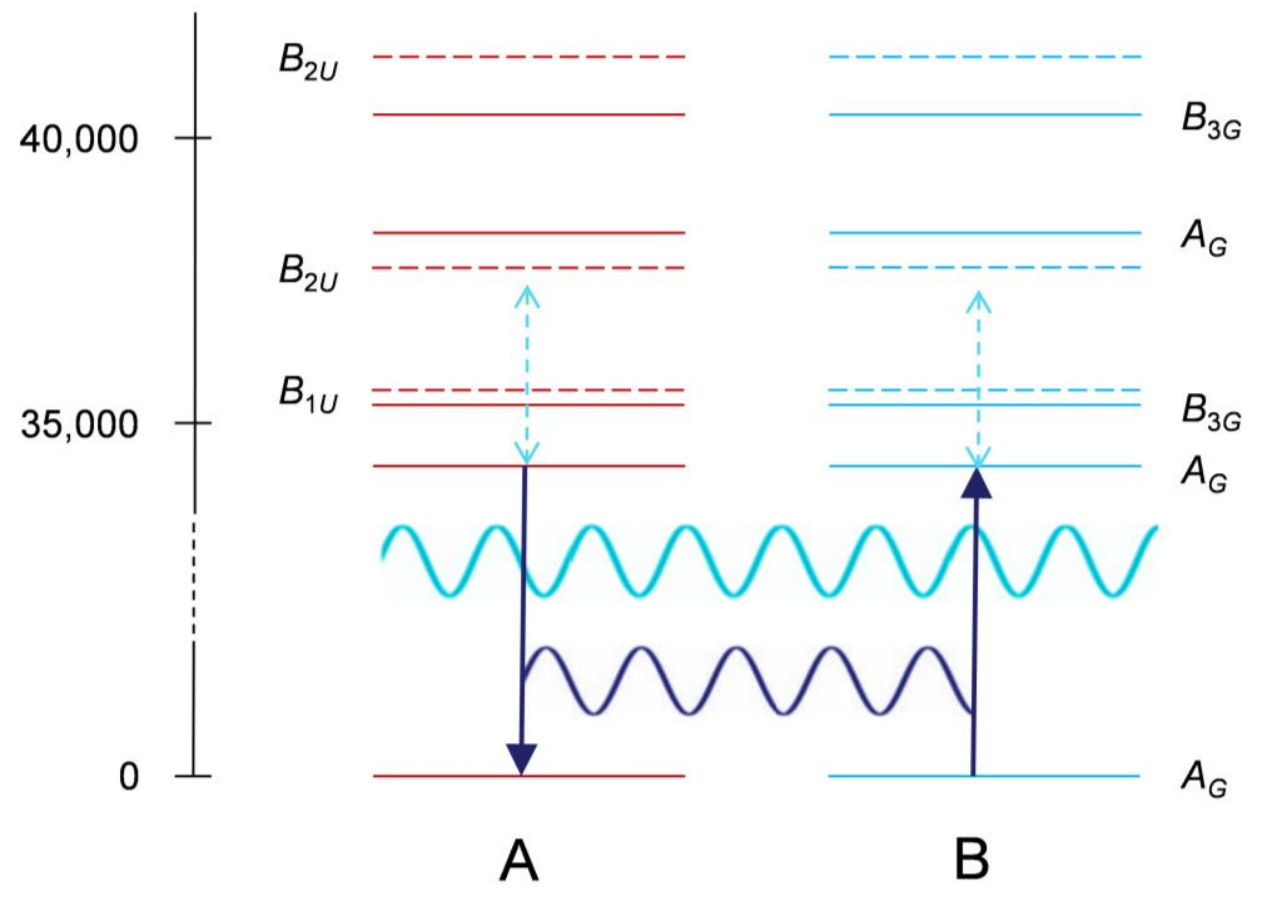

Fig. 2. Energy-level diagram representing OCRET between a donor porphine, $A$, and an acceptor, $B$. Horizontal solid and dashed lines denote energy levels corresponding to one-photon forbidden and allowed states, respectively; the symmetry of each energy level is also presented, and the energy scale on the left-hand side is given in wavenumbers $\left(\mathrm{cm}^{-1}\right)$. Solid vertical arrows signify real (representative) electronic transitions, the double-headed dashed arrows denote typical virtual transitions due to the control beam; the upper and lower wavy lines denote the control beam and the energy transfer, respectively. The OCRET mechanism is shown operating between the lowest dark (one-photon forbidden) states; calculations are also performed on transitions between other dark states.

efficiencies have already been determined for a variety of systems constructed in the form of nanoscale arrays, and configured to provide parallel signal-processing capability. ${ }^{8,9}$ In all of this earlier work, the detailed expressions for the efficiency of energy transfer were secured by QED analysis.

The OCRET mechanism involves simultaneous coupling between the off-resonant laser beam, and energy transfer from an excited donor $A$ to an acceptor $B$ (in its ground state); this is illustrated by the 
energy scheme of Fig. 2 and involves four fundamental light-molecule interactions. To specifically determine the rate of OCRET from $A$ to $B$, a time-dependent perturbation method is required; the detailed procedure of which, deployed to establish the quantum amplitudes of the system, is described elsewhere. ${ }^{10}$ In the near-field, over which the strength of the coupling mechanism is largest and the sought effect therefore most prominent, the quantum amplitude for OCRET is given by; ${ }^{8}$

$$
M_{f i}=\left(\frac{I}{2 \varepsilon_{0} c}\right) e_{i} \bar{e}_{l} V_{j k}(0, \mathbf{R})\left(S_{i j}^{A}(k) S_{l k}^{B}(-k)+S_{i j}^{B}(k) S_{l k}^{A}(-k)\right),
$$

where $I$ is the intensity of the input beam, $V_{j k}(0, \mathbf{R})$ represents the near-field E1-E1 coupling tensor ${ }^{11}$ ( $\mathbf{R}$ being the displacement vector between $A$ and $B$ ), and the implied summation convention for repeated Cartesian tensor indices is employed. Furthermore, e and $\hbar c k$ denote the polarization vector (an overbar denoting complex conjugation) and energy of a photon from the input laser, respectively; $S_{i j}^{\xi}( \pm k)$ is the generalized transition polarizability, written explicitly as;

$$
S_{i j}^{\xi}( \pm k)=\sum_{r}\left\{\frac{\mu_{i}^{f r} \mu_{j}^{r i}}{\tilde{E}_{r f} \pm \hbar c k}+\frac{\mu_{j}^{f r} \mu_{i}^{r i}}{\tilde{E}_{r i} \mp \hbar c k}\right\}
$$

where the transition dipole moments are designated by the shorthand notation $\boldsymbol{\mu}^{x y}=\langle x|\boldsymbol{\mu}| y\rangle$, energy differences $E_{x y}=E_{x}-E_{y}$, and tildes denote the inclusion of a damping term $\mathrm{i} \gamma$, where $2 \gamma$ is the FWHM linewidth. Further, $f$ signifies the final electronic state of molecule $\xi$ (either $A$ or $B$ ), $i$ the initial state and $r, s$ are intermediates. An expression to determine the rate, $\Gamma$, of OCRET is delivered from Fermi's Golden Rule, ${ }^{12}$ namely $\Gamma=2 \pi \rho_{f}\left|M_{f i}\right|^{2} / \hbar$ where $\rho_{f}$ is the density of states. 
Calculations on specific molecular systems have been performed using the Gaussian 03 computational package. ${ }^{13}$ In all calculations on the porphine molecules a point symmetry group of $D_{2 h}$ has been employed. The ground state geometry of porphine was optimized (Fig. 1) using the three parameter exchange functional of Becke ${ }^{14}$ (B3) and the correlation functional of Lee, Yang, and Parr ${ }^{15}$ (LYP), B3LYP with the triple-zeta split valence basis set, $6-311+G(d, p) .{ }^{14,16,17}$ The chromophores were constrained to $D_{2 h}$ symmetry and confirmed as minima through frequency analysis, and in each case these structures were used throughout the excited state calculations. The electronic excited states were investigated using the symmetry-adapted-cluster ${ }^{18}$ (SAC)/configuration interaction ${ }^{19-21}(\mathrm{CI})$ method with the double-zeta split valence basis set, $6-31+\mathrm{G}(\mathrm{d}, \mathrm{p}) .{ }^{14,16,17}$ For each irreducible symmetry representation all singlet $\rightarrow$ singlet multi-electron excited states with energies up to $11 \mathrm{eV}$ (total of 98 , including the ground state) were calculated using an active space comprising the 7 highest occupied and 7 lowest virtual orbitals. The associated transition dipole moments and excitation energies were calculated directly from SAC/CI results. The values of the energies and oscillator strengths for the symmetryallowed electronic transitions of the porphine macrocycle calculated by SAC-CI approach correspond well to those calculated by other $a b$ initio methods ${ }^{22-23}$ and are in good agreement with the experimental observations. ${ }^{22,24}$ To the best of our knowledge, this calculation of transition dipole matrix elements among excited states of porphine molecule by the SAC-CI method has been carried out for the first time.

A computer simulation program has been developed to enable calculation of the OCRET rates from $a b$ initio results in accordance with equations (1) and (2). These rates can then be determined for variable frequencies and polarizations of the input laser beam, here assuming an irradiance of $5 \times 10^{11}$ $\mathrm{W} \mathrm{cm} \mathrm{cm}^{-2}$, for both the stacked and co-planar configurations. For the purposes of this work, electronic transitions within the polycyclic (porphine) chromophores are chosen so that one-photon transitions are forbidden by symmetry selection rules, i.e. dark states are selected. Therefore, Förster (or transitiondipole mediated) transfer involving these states is excluded, but the two-photon transitions of OCRET 
are allowed. The resonance damping factor is arbitrarily fixed at $200 \mathrm{~cm}^{-1}$, sufficient to generate a realistic line-shape without producing overlapped electronic states. The values for each excited state will in fact differ, but those values are unknown and they are not amenable to calculation. In fact, the reported effects and the conclusions to be drawn are not especially sensitive to the precise values of the damping constant. Employing equation (1) and Fermi's Golden Rule, it is convenient to determine ratios of the OCRET rate with respect to the rate of Förster transfer between the lowest one-photon allowed states of the chromophore. These ratios have been determined over the frequency range $(1 \times$ $\left.10^{4}-4 \times 10^{4}\right) \mathrm{cm}^{-1}$, and for the input laser polarized in the $\mathrm{X}, \mathrm{Y}$ or $\mathrm{Z}$ direction. In detail, the employed dark states of porphine are those at the lowest energies above the ground state: $33945 \mathrm{~cm}^{-1}$ (state 1 , symmetry $\left.A_{G}\right), 35192 \mathrm{~cm}^{-1}\left(2, B_{3 G}\right), 38195 \mathrm{~cm}^{-1}\left(3, A_{G}\right), 40132 \mathrm{~cm}^{-1}\left(4, B_{3 G}\right), 43129 \mathrm{~cm}^{-1}\left(5, B_{3 G}\right)$, and $43866 \mathrm{~cm}^{-1}\left(6, A_{G}\right)$. The lowest accessible Förster allowed energy level is found at $35456 \mathrm{~cm}^{-1}\left(B_{1 U}\right)$.

Following calculation of the OCRET rates from the simulation program, plots of the ratios against laser frequency are constructed; the most significant of which are shown in Fig. 3. Since the phenomenon of interest designedly deploys an off-resonant laser beam, any results for conditions 'on' or approaching resonance are omitted. Although larger ratios can be secured by resonance enhancement, the chromophore system must remain transparent with respect to the input beam for experimentally meaningful results. The calculations are determined in accordance with the selection rules imposed by $D_{2 h}$ symmetry, and only transitions involving dark states are OCRET active.

From the constructed graphs, the most interesting aspects are that the ratios are: (i) widely varying with input frequency, because of the dependence on the fourth power of the transition polarizability; (ii) crucially dependent on dispersion behavior; (iii) negligible for an X polarized input laser. Analysis of 


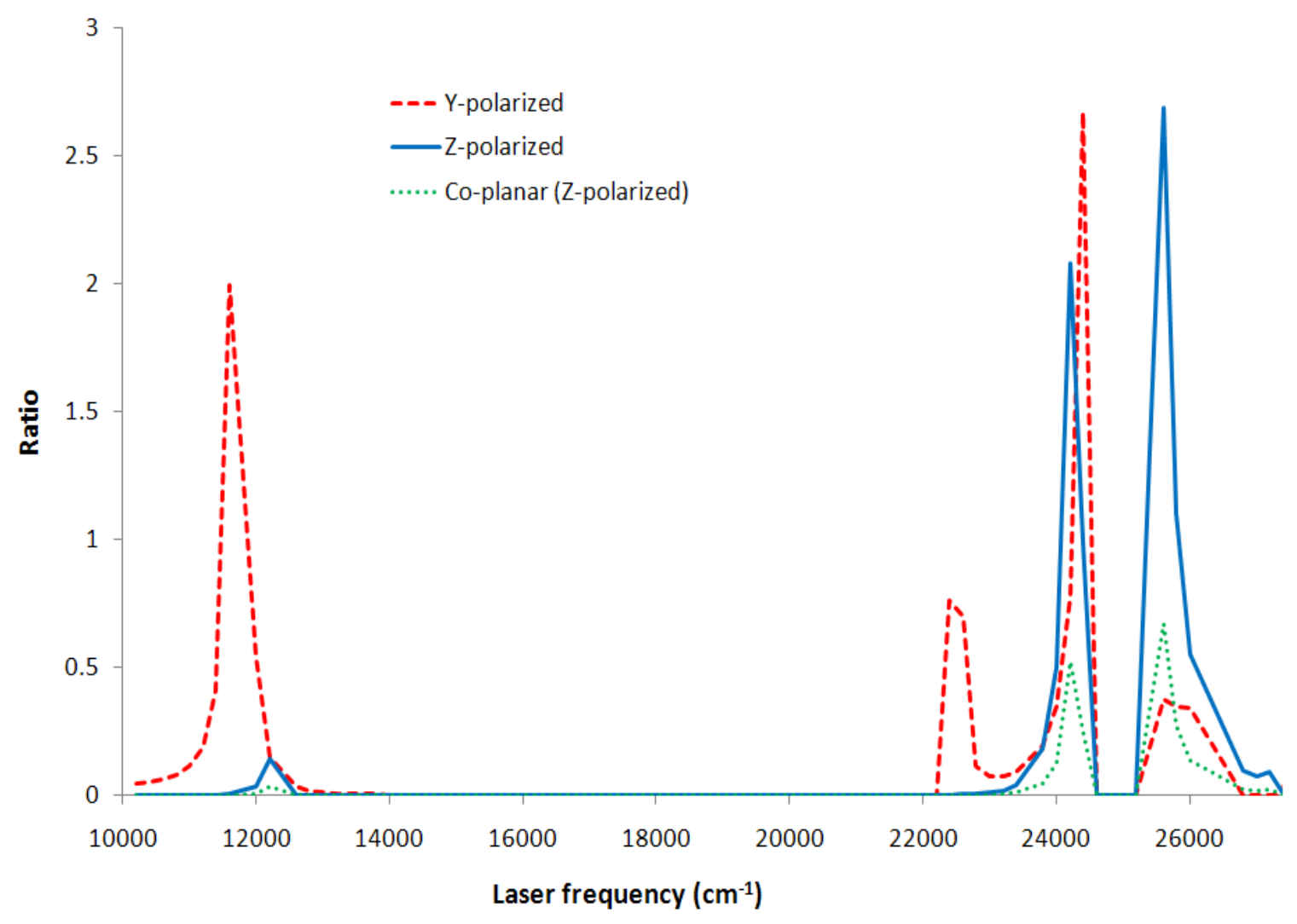

Fig. 3. Graph of the OCRET versus Förster rate, i.e. the ratio described in the text, against input laser frequency for the stacked configuration (unless stated). Here, the lowest dark state (state 1, symmetry $\left.A_{G}\right)$ is employed in the OCRET calculations and results very close to resonance are omitted, as seen between 24000 and $26000 \mathrm{~cm}^{-1}$; except omitted results, none of the ratios are exactly zero.

the results from ab initio calculations has shown that multi-electron excited states $B_{3 u}$ have relatively high excitation energies $(>7 \mathrm{eV})$ and, therefore, are well separated from both the ground state and excited states $A_{G}(1,3,6)$ and $B_{3 G}(2,4,5)$. This ensures that the contributions from the intermediate states $B_{3 u}$ (via the symmetry allowed non-zero X-components of the transition dipole moment with ground and/or excited states) into the relevant generalized transition polarizability tensor components $S_{x x}, S_{x y}, S_{x z}, S_{y x}, S_{z x}$ are weak. The relative position of all intermediate excited states have a profound effect on the calculated properties because of the dependence on the fourth power of the transition polarizability; (iv) often dissimilar for Y polarized light compared to $\mathrm{Z}$ polarized input with matching 
laser frequencies; (v) identical for $A_{G}$ dark states and $\mathrm{Y}$ polarized light whether the stacked and coplanar configurations is applied. This is again a consequence of the selection rules imposed by the symmetry on the generalized transition polarizability tensor via transition dipole matrix elements. For excited states $A_{G}$ only $V_{y y}(0, R) e_{y} S_{y y}^{A} S_{y y}^{B} e_{y}$ term has non-zero contribution into $M_{f i}$. Since $V_{y y}(0, R)$ does not change upon changing the configuration from stack to co-planar the ratios are identical; (vi) also identical for $B_{3 G}$ dark states and $\mathrm{Z}$ polarized light. In this case the non-zero contributing term is $V_{y y}(0, R) e_{z} S_{z y}^{A} S_{z y}^{B} e_{z}$ and, therefore, an analogous conclusion about the ratios can be drawn; (vii) enhanced for $A_{G}$ states and $\mathrm{Z}$ polarized light comparing the stacked configuration against the co-planar. This is because the only non-zero terms allowed by symmetry, namely $V_{z z}(0, R) e_{z} S_{z z}^{A} S_{z z}^{B} e_{z}$ depend on the $V_{z z}(0, R)$ component of the near-field E1-E1 coupling tensor. The absolute value of this component is decreased from stacked to co-planar configuration; (viii) also enhanced for $B_{3 G}$ states and Y polarized light. Here the non-zero contributions come from $V_{z z}(0, R) e_{y} S_{y z}^{A} S_{y z}^{B} e_{y}$ terms and therefore similar arguments apply. It is noteworthy that, in terms of the absolute rate, the stacked configuration will likely be favored over the co-planar case, since the former allows closer spacing of the ring structures. For example, since the absolute rate is dependent on $R^{-6}$, a factor of $\sim 3$ difference in the separation translates into an approximately three orders of magnitude difference in the rates.

In summary, we report the first quantitative prediction of OCRET rates from ab initio calculations. The energy transfer rates have been calculated and compared for two configurations of chromophores, namely, stacked and co-planar. It is shown that, for porphine molecules, OCRET (a two-photon allowed mechanism) may be comparable in efficiency to the conventional Foster transfer mechanism, and therefore should be experimentally detectable. The predicted OCRET rates show strong dependence on the polarization of the laser beam; this and other observations are predetermined by the symmetry of the chromophore. The OCRET mechanism occurs via dark (one-photon forbidden by symmetry) states in contrast with the Foster transfer which is transition dipole mediated. The former mechanism promises 
the benefit of direct optical control over the energy transfer. The ability to predict transfer efficiencies from the state-of-the-art ab initio calculations of real molecular structures promises to facilitate the design of experimental schemes utilizing OCRET.

Acknowledgement This work is financially supported by the Leverhulme Trust (DSB, DLA).

\section{References}

1. Craig, D. P.; Thirunamachandran, T. Molecular Quantum Electrodynamics; Dover: Mineola, N.Y., 1998.

2. Andrews, D. L.; Allcock, P. Optical Harmonics in Molecular Systems; Wiley-VCH: Weinheim, Germany, 2002.

3. Salam, A. Molecular Quantum Electrodynamics: Long-Range Intermolecular Interactions; Wiley: Hoboken, N.J., 2010.

4. Scholes, G. D. Long-Range Resonance Energy Transfer in Molecular Systems, Annu. Rev. Phys. Chem. 2003, 54, 57-87.

5. Richter, M.; Ahn K. J.; Knorr, A.; Schliwa, A.; Bimberg, D.; Madjet, M. E.-A.; Renger, T. Theory of Excitation Transfer in Coupled Nanostructures - From Quantum Dots to Light Harvesting Complexes, Phys. Stat. Sol. (b) 2006, 243, 2302-2310.

6. Andrews, D. L.; Bradshaw, D. S. In Encyclopedia of Applied Spectroscopy; Andrews, D. L., Ed.; Wiley-VCH: Weinheim, Germany, 2009; pp 533-554. 
7. Andrews, D. L.; Crisp, R. G. Optically Activated Energy Transfer: Array Implementation, J. Opt. A: Pure Appl. Opt. 2006, 8, S106-S112.

8. Bradshaw, D. S.; Andrews, D. L. Optically Controlled Resonance Energy Transfer: Mechanism and Configuration for All-Optical Switching, J. Chem. Phys. 2008, 128, 144506.

9. Bradshaw, D. S.; Andrews, D. L. All-Optical Switching Between Quantum Dot Nanoarrays, Superlatt. Microstruct. 2010, 47, 308-313.

10. Allcock, P.; Jenkins, R. D.; Andrews, D. L. Laser-Assisted Resonance-Energy Transfer, Phys. Rev. A 2000, 61, 023812.

11. Andrews, D. L.; Bradshaw, D. S. Virtual Photons, Dipole Fields and Energy Transfer: A Quantum Electrodynamical Approach, Eur. J. Phys. 2004, 25, 845-858.

12. Mandel, L.; Wolf, E. Optical Coherence and Quantum Optics; University Press: Cambridge, U.K., 1995, p. 871.

13. Frisch, M. J.; Trucks, G. W.; Schlegel, H. B.; Scuseria, G. E.; Robb, M. A.; Cheeseman, J. R.; Montgomery, J. A., Jr.; Vreven, T.; Kudin, K. N.; Burant, J. C.; et al. Gaussian 03, Revision E.01; Gaussian, Inc., Wallingford CT, 2004.

14. Becke, A. D. Density-Functional Thermochemistry. III. The Role of Exact Exchange, J. Chem. Phys. 1993, 98, 5648-5652.

15. Lee, C.; Yang, W.; Parr, R. G. Development of the Colle-Salvetti Correlation-Energy Formula into a Functional of the Electron Density, Phys. Rev. B: Condens. Matter. 1988, 37, 785-789.

16. Poirer, R.; Kari, R.; Csizmadia, I. G. Handbook of Gaussian Basis Sets: A Compendium for Ab Initio Molecular Orbital Calculations; Elsevier Science Publishing: New York, 1985.

17. Huzinaga, S. (ed.) Gaussian Basis Sets for Molecular Calculations; Elsevier: Amsterdam, 1984. 
18. Nakatsuji, H.; Hirao, K. Cluster Expansion of the Wavefunction. Symmetry-Adapted-Cluster Expansion, its Variational Determination, and Extension of Open-Shell Orbital Theory, J. Chem. Phys. 1978, 68, 2053-2065.

19. Nakatsuji, H. Cluster Expansion of the Wavefunction. Excited States, Chem. Phys. Lett. 1978, 59, 362-364.

20. Nakatsuji, H. Cluster Expansion of the Wavefunction. Electron Correlations in Ground and Excited States by SAC (Symmetry-Adapted-Cluster) and SAC CI Theories, Chem. Phys. Lett. 1979, 67, 329-333.

21. Nakatsuji, H. Cluster Expansion of the Wavefunction. Calculation of Electron Correlations in Ground and Excited States by SAC and SAC CI Theories, Chem. Phys. Lett. 1979, 67, 334-342.

22. Moroni, L.; Gellini, C.; Salvi, P. R.; Marcelli, A.; Foggi P. Excited States of Porphyrin Molecules, J. Phys. Chem. A 2008, 112, 11044-11051.

23. Serrano-Andres, L.; Merchan, M.; Rubio, M.; Roos, B. O. Interpretation of the Electronic Absorption Spectrum of Free Base Porphin by using Multiconfigurational Second-Order Perturbation Theory, Chem. Phys. Lett. 1998, 295, 195-203.

24. Nagashima, U.; Takada, T.; Ohno, K. Ab Initio SCF-CL Calculation on Free Base Porphin and Chlorine - Theoretical Analysis and Intensities of the Absorption Spectra, J. Chem. Phys. 1986, $85,4524-4529$. 


\section{Supporting Information Available}

Excitation energies, components of the transition dipole moments and oscillator strengths between the singlet excited states and the ground state as calculated by symmetry-adapted-cluster (SAC) / configuration interaction (CI) method:

(D2h symmetry)

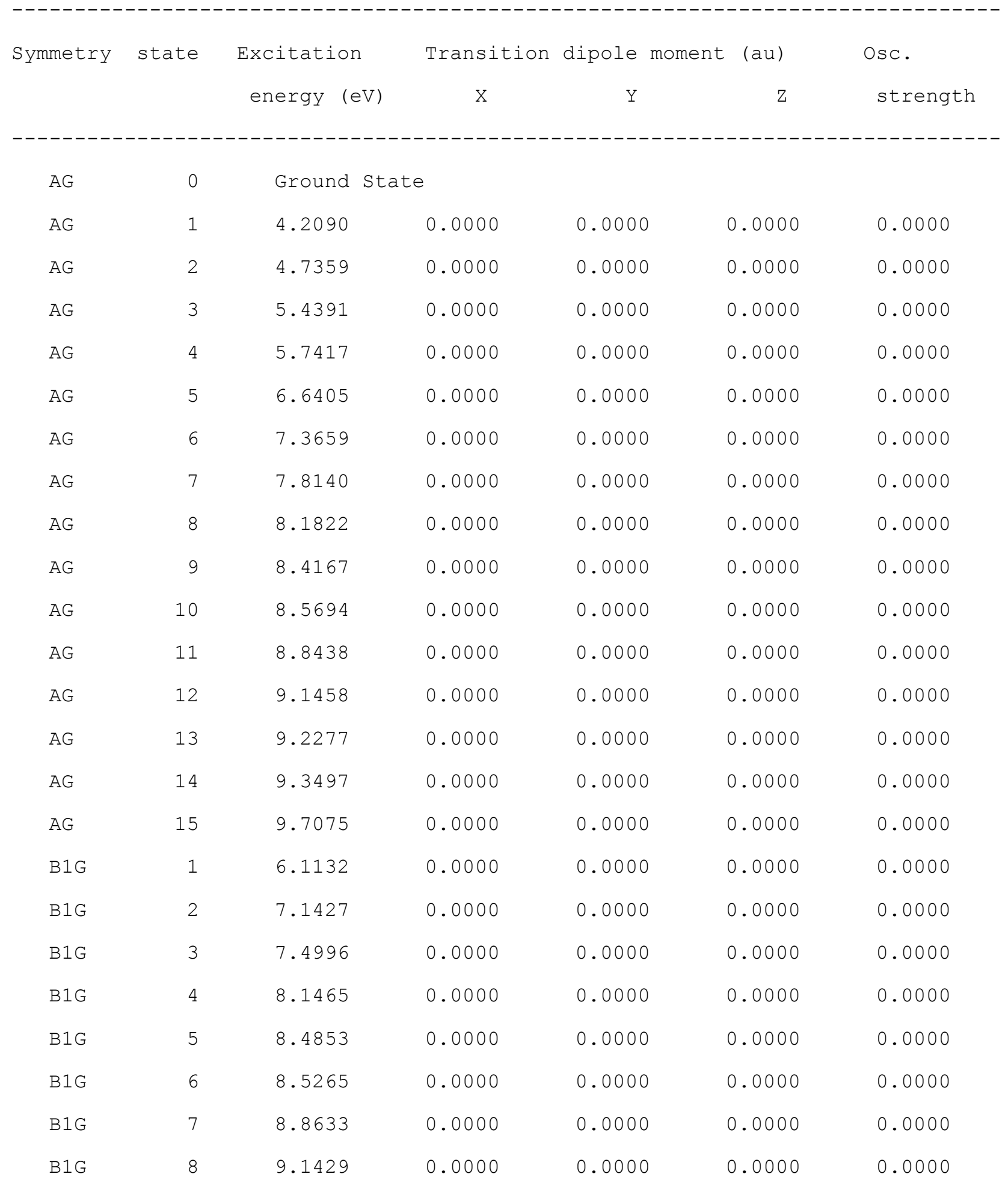




\begin{tabular}{|c|c|c|c|c|c|c|}
\hline $\mathrm{B} 1 \mathrm{G}$ & 9 & 9.4472 & 0.0000 & 0.0000 & 0.0000 & 0.0000 \\
\hline $\mathrm{B} 1 \mathrm{G}$ & 10 & 9.8505 & 0.0000 & 0.0000 & 0.0000 & 0.0000 \\
\hline B2G & 1 & 6.2584 & 0.0000 & 0.0000 & 0.0000 & 0.0000 \\
\hline B2G & 2 & 6.9305 & 0.0000 & 0.0000 & 0.0000 & 0.0000 \\
\hline B2G & 3 & 7.8565 & 0.0000 & 0.0000 & 0.0000 & 0.0000 \\
\hline B2G & 4 & 8.3250 & 0.0000 & 0.0000 & 0.0000 & 0.0000 \\
\hline B2G & 5 & 8.5910 & 0.0000 & 0.0000 & 0.0000 & 0.0000 \\
\hline B2G & 6 & 8.7956 & 0.0000 & 0.0000 & 0.0000 & 0.0000 \\
\hline B2G & 7 & 9.1467 & 0.0000 & 0.0000 & 0.0000 & 0.0000 \\
\hline B2G & 8 & 9.2626 & 0.0000 & 0.0000 & 0.0000 & 0.0000 \\
\hline B2G & 9 & 9.3426 & 0.0000 & 0.0000 & 0.0000 & 0.0000 \\
\hline B2G & 10 & 10.2693 & 0.0000 & 0.0000 & 0.0000 & 0.0000 \\
\hline B3G & 1 & 4.3636 & 0.0000 & 0.0000 & 0.0000 & 0.0000 \\
\hline B3G & 2 & 4.9761 & 0.0000 & 0.0000 & 0.0000 & 0.0000 \\
\hline B3G & 3 & 5.3477 & 0.0000 & 0.0000 & 0.0000 & 0.0000 \\
\hline B3G & 4 & 6.6174 & 0.0000 & 0.0000 & 0.0000 & 0.0000 \\
\hline B3G & 5 & 7.4542 & 0.0000 & 0.0000 & 0.0000 & 0.0000 \\
\hline B3G & 6 & 7.7158 & 0.0000 & 0.0000 & 0.0000 & 0.0000 \\
\hline B3G & 7 & 7.8191 & 0.0000 & 0.0000 & 0.0000 & 0.0000 \\
\hline B3G & 8 & 7.9546 & 0.0000 & 0.0000 & 0.0000 & 0.0000 \\
\hline B3G & 9 & 8.3682 & 0.0000 & 0.0000 & 0.0000 & 0.0000 \\
\hline B3G & 10 & 8.4880 & 0.0000 & 0.0000 & 0.0000 & 0.0000 \\
\hline B3G & 11 & 8.6908 & 0.0000 & 0.0000 & 0.0000 & 0.0000 \\
\hline B3G & 12 & 8.7864 & 0.0000 & 0.0000 & 0.0000 & 0.0000 \\
\hline B3G & 13 & 9.0055 & 0.0000 & 0.0000 & 0.0000 & 0.0000 \\
\hline B3G & 14 & 9.3014 & 0.0000 & 0.0000 & 0.0000 & 0.0000 \\
\hline B3G & 15 & 9.8903 & 0.0000 & 0.0000 & 0.0000 & 0.0000 \\
\hline $\mathrm{AU}$ & 1 & 5.8268 & 0.0000 & 0.0000 & 0.0000 & 0.0000 \\
\hline$A U$ & 2 & 6.4729 & 0.0000 & 0.0000 & 0.0000 & 0.0000 \\
\hline AU & 3 & 8.2561 & 0.0000 & 0.0000 & 0.0000 & 0.0000 \\
\hline $\mathrm{AU}$ & 4 & 8.8727 & 0.0000 & 0.0000 & 0.0000 & 0.0000 \\
\hline $\mathrm{AU}$ & 5 & 9.1009 & 0.0000 & 0.0000 & 0.0000 & 0.0000 \\
\hline $\mathrm{AU}$ & 6 & 9.1643 & 0.0000 & 0.0000 & 0.0000 & 0.0000 \\
\hline $\mathrm{AU}$ & 7 & 9.3071 & 0.0000 & 0.0000 & 0.0000 & 0.0000 \\
\hline $\mathrm{AU}$ & 8 & 10.3116 & 0.0000 & 0.0000 & 0.0000 & 0.0000 \\
\hline
\end{tabular}




\begin{tabular}{|c|c|c|c|c|c|c|}
\hline $\mathrm{B} 1 \mathrm{U}$ & 1 & 3.1464 & 0.0000 & 0.0000 & 0.4054 & 0.0127 \\
\hline $\mathrm{B} 1 \mathrm{U}$ & 2 & 4.3960 & 0.0000 & 0.0000 & 0.1755 & 0.0033 \\
\hline $\mathrm{B} 1 \mathrm{U}$ & 3 & 5.6616 & 0.0000 & 0.0000 & 0.1311 & 0.0024 \\
\hline $\mathrm{B} 1 \mathrm{U}$ & 4 & 6.1606 & 0.0000 & 0.0000 & -0.4535 & 0.0310 \\
\hline $\mathrm{B} 1 \mathrm{U}$ & 5 & 6.8263 & 0.0000 & 0.0000 & -0.0993 & 0.0017 \\
\hline $\mathrm{B} 1 \mathrm{U}$ & 6 & 7.8002 & 0.0000 & 0.0000 & -0.0008 & 0.0000 \\
\hline $\mathrm{B} 1 \mathrm{U}$ & 7 & 8.2199 & 0.0000 & 0.0000 & 0.0767 & 0.0012 \\
\hline $\mathrm{B} 1 \mathrm{U}$ & 8 & 8.7194 & 0.0000 & 0.0000 & 0.0145 & 0.0000 \\
\hline $\mathrm{B} 1 \mathrm{U}$ & 9 & 8.8638 & 0.0000 & 0.0000 & -0.4269 & 0.0396 \\
\hline $\mathrm{B} 1 \mathrm{U}$ & 10 & 9.1842 & 0.0000 & 0.0000 & 0.2740 & 0.0169 \\
\hline $\mathrm{B} 1 \mathrm{U}$ & 11 & 9.3073 & 0.0000 & 0.0000 & -0.0344 & 0.0003 \\
\hline $\mathrm{B} 1 \mathrm{U}$ & 12 & 9.3611 & 0.0000 & 0.0000 & -0.2273 & 0.0118 \\
\hline $\mathrm{B} 1 \mathrm{U}$ & 13 & 9.4423 & 0.0000 & 0.0000 & -0.5233 & 0.0633 \\
\hline $\mathrm{B} 1 \mathrm{U}$ & 14 & 9.8502 & 0.0000 & 0.0000 & -0.0275 & 0.0002 \\
\hline $\mathrm{B} 1 \mathrm{U}$ & 15 & 10.0371 & 0.0000 & 0.0000 & -0.0522 & 0.0007 \\
\hline $\mathrm{B} 2 \mathrm{U}$ & 1 & 3.4108 & 0.0000 & 0.3585 & 0.0000 & 0.0107 \\
\hline $\mathrm{B} 2 \mathrm{U}$ & 2 & 4.6973 & 0.0000 & -0.1721 & 0.0000 & 0.0034 \\
\hline $\mathrm{B} 2 \mathrm{U}$ & 3 & 5.1424 & 0.0000 & -0.0408 & 0.0000 & 0.0002 \\
\hline $\mathrm{B} 2 \mathrm{U}$ & 4 & 6.0624 & 0.0000 & -0.3828 & 0.0000 & 0.0218 \\
\hline $\mathrm{B} 2 \mathrm{U}$ & 5 & 7.7692 & 0.0000 & 0.0666 & 0.0000 & 0.0008 \\
\hline $\mathrm{B} 2 \mathrm{U}$ & 6 & 7.8968 & 0.0000 & -0.1327 & 0.0000 & 0.0034 \\
\hline $\mathrm{B} 2 \mathrm{U}$ & 7 & 8.3889 & 0.0000 & 0.1068 & 0.0000 & 0.0023 \\
\hline $\mathrm{B} 2 \mathrm{U}$ & 8 & 8.5656 & 0.0000 & -0.0171 & 0.0000 & 0.0001 \\
\hline $\mathrm{B} 2 \mathrm{U}$ & 9 & 8.8415 & 0.0000 & -0.0941 & 0.0000 & 0.0019 \\
\hline $\mathrm{B} 2 \mathrm{U}$ & 10 & 9.2252 & 0.0000 & 0.2288 & 0.0000 & 0.0118 \\
\hline $\mathrm{B} 2 \mathrm{U}$ & 11 & 9.3314 & 0.0000 & -0.7020 & 0.0000 & 0.1127 \\
\hline B2U & 12 & 9.7396 & 0.0000 & 0.0766 & 0.0000 & 0.0014 \\
\hline B2U & 13 & 9.8856 & 0.0000 & -0.1398 & 0.0000 & 0.0047 \\
\hline $\mathrm{B} 2 \mathrm{U}$ & 14 & 10.0245 & 0.0000 & 0.0123 & 0.0000 & 0.0000 \\
\hline B3U & 1 & 7.4782 & 0.0448 & 0.0000 & 0.0000 & 0.0004 \\
\hline B3U & 2 & 7.8378 & 0.0820 & 0.0000 & 0.0000 & 0.0013 \\
\hline B3U & 3 & 8.2424 & -0.0248 & 0.0000 & 0.0000 & 0.0001 \\
\hline B3U & 4 & 8.4987 & 0.0041 & 0.0000 & 0.0000 & 0.0000 \\
\hline B3U & 5 & 8.6466 & 0.0025 & 0.0000 & 0.0000 & 0.0000 \\
\hline B3U & 6 & 8.7867 & 0.0094 & 0.0000 & 0.0000 & 0.0000 \\
\hline
\end{tabular}




$\begin{array}{lrrrrrr}\text { B3U } & 7 & 8.8206 & 0.0279 & 0.0000 & 0.0000 & 0.0002 \\ \text { B3U } & 8 & 8.8624 & -0.0450 & 0.0000 & 0.0000 & 0.0004 \\ \text { B3U } & 9 & 9.0311 & 0.0097 & 0.0000 & 0.0000 & 0.0000 \\ \text { B3U } & 10 & 9.0918 & -0.0094 & 0.0000 & 0.0000 & 0.0000\end{array}$

Representative table showing the transition dipole moments, oscillator strengths and relative energies between the lowest dark state (state 1 , symmetry $A_{G}$, excitation energy $=4.2090 \mathrm{eV}\left(33945 \mathrm{~cm}^{-1}\right)$ ) and the rest of the excited states used in the calculations. These data have been used for the calculation of OCRET rates and their comparison with the Förster rate as presented in Figure 3.

\begin{tabular}{|c|c|c|c|c|c|c|}
\hline \multirow[t]{2}{*}{ Symmetry } & \multirow[t]{2}{*}{ State } & relative & \multicolumn{3}{|c|}{ Transition dipole moment } & \multirow{2}{*}{$\begin{array}{c}\text { Osc. } \\
\text { strength }\end{array}$} \\
\hline & & energy (eV) & $\mathrm{X}$ & Y & $\mathrm{Z}$ & \\
\hline AG & 1 & 0.0 & Excited & State 1 . & & \\
\hline AG & 2 & 0.5269 & 0.0000 & 0.0000 & 0.0000 & 0.0000 \\
\hline AG & 3 & 1.2301 & 0.0000 & 0.0000 & 0.0000 & 0.0000 \\
\hline AG & 4 & 1.5327 & 0.0000 & 0.0000 & 0.0000 & 0.0000 \\
\hline AG & 5 & 2.4314 & 0.0000 & 0.0000 & 0.0000 & 0.0000 \\
\hline$A G$ & 6 & 3.1568 & 0.0000 & 0.0000 & 0.0000 & 0.0000 \\
\hline AG & 7 & 3.6050 & 0.0000 & 0.0000 & 0.0000 & 0.0000 \\
\hline AG & 8 & 3.9731 & 0.0000 & 0.0000 & 0.0000 & 0.0000 \\
\hline$A G$ & 9 & 4.2076 & 0.0000 & 0.0000 & 0.0000 & 0.0000 \\
\hline AG & 10 & 4.3604 & 0.0000 & 0.0000 & 0.0000 & 0.0000 \\
\hline$A G$ & 11 & 4.6348 & 0.0000 & 0.0000 & 0.0000 & 0.0000 \\
\hline AG & 12 & 4.9367 & 0.0000 & 0.0000 & 0.0000 & 0.0000 \\
\hline AG & 13 & 5.0187 & 0.0000 & 0.0000 & 0.0000 & 0.0000 \\
\hline AG & 14 & 5.1406 & 0.0000 & 0.0000 & 0.0000 & 0.0000 \\
\hline$A G$ & 15 & 5.4985 & 0.0000 & 0.0000 & 0.0000 & 0.0000 \\
\hline B1G & 1 & 1.9041 & 0.0000 & 0.0000 & 0.0000 & 0.0000 \\
\hline $\mathrm{B} 1 \mathrm{G}$ & 2 & 2.9336 & 0.0000 & 0.0000 & 0.0000 & 0.0000 \\
\hline $\mathrm{B} 1 \mathrm{G}$ & 3 & 3.2906 & 0.0000 & 0.0000 & 0.0000 & 0.0000 \\
\hline B1G & 4 & 3.9374 & 0.0000 & 0.0000 & 0.0000 & 0.0000 \\
\hline
\end{tabular}




\begin{tabular}{|c|c|c|c|c|c|c|}
\hline $\mathrm{B} 1 \mathrm{G}$ & 5 & 4.2763 & 0.0000 & 0.0000 & 0.0000 & 0.0000 \\
\hline $\mathrm{B} 1 \mathrm{G}$ & 6 & 4.3174 & 0.0000 & 0.0000 & 0.0000 & 0.0000 \\
\hline $\mathrm{B} 1 \mathrm{G}$ & 7 & 4.6542 & 0.0000 & 0.0000 & 0.0000 & 0.0000 \\
\hline $\mathrm{B} 1 \mathrm{G}$ & 8 & 4.9338 & 0.0000 & 0.0000 & 0.0000 & 0.0000 \\
\hline $\mathrm{B} 1 \mathrm{G}$ & 9 & 5.2382 & 0.0000 & 0.0000 & 0.0000 & 0.0000 \\
\hline $\mathrm{B} 1 \mathrm{G}$ & 10 & 5.6417 & 0.0000 & 0.0000 & 0.0000 & 0.0000 \\
\hline B2G & 1 & 2.0493 & 0.0000 & 0.0000 & 0.0000 & 0.0000 \\
\hline B2G & 2 & 2.7215 & 0.0000 & 0.0000 & 0.0000 & 0.0000 \\
\hline B2G & 3 & 3.6474 & 0.0000 & 0.0000 & 0.0000 & 0.0000 \\
\hline B2G & 4 & 4.1159 & 0.0000 & 0.0000 & 0.0000 & 0.0000 \\
\hline B2G & 5 & 4.3820 & 0.0000 & 0.0000 & 0.0000 & 0.0000 \\
\hline B2G & 6 & 4.5866 & 0.0000 & 0.0000 & 0.0000 & 0.0000 \\
\hline B2G & 7 & 4.9377 & 0.0000 & 0.0000 & 0.0000 & 0.0000 \\
\hline B2G & 8 & 5.0536 & 0.0000 & 0.0000 & 0.0000 & 0.0000 \\
\hline B2G & 9 & 5.1335 & 0.0000 & 0.0000 & 0.0000 & 0.0000 \\
\hline B2G & 10 & 6.0603 & 0.0000 & 0.0000 & 0.0000 & 0.0000 \\
\hline B3G & 1 & 0.1546 & 0.0000 & 0.0000 & 0.0000 & 0.0000 \\
\hline B3G & 2 & 0.7670 & 0.0000 & 0.0000 & 0.0000 & 0.0000 \\
\hline B3G & 3 & 1.1387 & 0.0000 & 0.0000 & 0.0000 & 0.0000 \\
\hline B3G & 4 & 2.4084 & 0.0000 & 0.0000 & 0.0000 & 0.0000 \\
\hline B3G & 5 & 3.2452 & 0.0000 & 0.0000 & 0.0000 & 0.0000 \\
\hline B3G & 6 & 3.5068 & 0.0000 & 0.0000 & 0.0000 & 0.0000 \\
\hline B3G & 7 & 3.6100 & 0.0000 & 0.0000 & 0.0000 & 0.0000 \\
\hline B3G & 8 & 3.7455 & 0.0000 & 0.0000 & 0.0000 & 0.0000 \\
\hline B3G & 9 & 4.1592 & 0.0000 & 0.0000 & 0.0000 & 0.0000 \\
\hline B3G & 10 & 4.2789 & 0.0000 & 0.0000 & 0.0000 & 0.0000 \\
\hline B3G & 11 & 4.4817 & 0.0000 & 0.0000 & 0.0000 & 0.0000 \\
\hline B3G & 12 & 4.5773 & 0.0000 & 0.0000 & 0.0000 & 0.0000 \\
\hline B3G & 13 & 4.7964 & 0.0000 & 0.0000 & 0.0000 & 0.0000 \\
\hline B3G & 14 & 5.0932 & 0.0000 & 0.0000 & 0.0000 & 0.0000 \\
\hline B3G & 15 & 5.6802 & 0.0000 & 0.0000 & 0.0000 & 0.0000 \\
\hline$A U$ & 1 & 1.6178 & 0.0000 & 0.0000 & 0.0000 & 0.0000 \\
\hline $\mathrm{AU}$ & 2 & 2.2638 & 0.0000 & 0.0000 & 0.0000 & 0.0000 \\
\hline $\mathrm{AU}$ & 3 & 4.0470 & 0.0000 & 0.0000 & 0.0000 & 0.0000 \\
\hline $\mathrm{AU}$ & 4 & 4.6637 & 0.0000 & 0.0000 & 0.0000 & 0.0000 \\
\hline
\end{tabular}




\begin{tabular}{|c|c|c|c|c|c|c|}
\hline$A U$ & 5 & 4.8919 & 0.0000 & 0.0000 & 0.0000 & 0.0000 \\
\hline$A U$ & 6 & 4.9552 & 0.0000 & 0.0000 & 0.0000 & 0.0000 \\
\hline $\mathrm{AU}$ & 7 & 5.0980 & 0.0000 & 0.0000 & 0.0000 & 0.0000 \\
\hline$A U$ & 8 & 6.1026 & 0.0000 & 0.0000 & 0.0000 & 0.0000 \\
\hline B1U & 1 & -1.0626 & 0.0000 & 0.0000 & -0.0486 & 0.0001 \\
\hline $\mathrm{B} 1 \mathrm{U}$ & 2 & 0.1870 & 0.0000 & 0.0000 & 0.4301 & 0.0008 \\
\hline $\mathrm{B} 1 \mathrm{U}$ & 3 & 1.4526 & 0.0000 & 0.0000 & -1.0072 & 0.0361 \\
\hline B1U & 4 & 1.9515 & 0.0000 & 0.0000 & 0.7034 & 0.0237 \\
\hline B1U & 5 & 2.6173 & 0.0000 & 0.0000 & 0.0082 & 0.0000 \\
\hline B1U & 6 & 3.5911 & 0.0000 & 0.0000 & 0.4027 & 0.0143 \\
\hline $\mathrm{B} 1 \mathrm{U}$ & 7 & 4.0109 & 0.0000 & 0.0000 & -0.0357 & 0.0001 \\
\hline $\mathrm{B} 1 \mathrm{U}$ & 8 & 4.5103 & 0.0000 & 0.0000 & 0.3227 & 0.0115 \\
\hline $\mathrm{B} 1 \mathrm{U}$ & 9 & 4.6547 & 0.0000 & 0.0000 & 0.1062 & 0.0013 \\
\hline B1U & 10 & 4.9752 & 0.0000 & 0.0000 & -0.1904 & 0.0044 \\
\hline $\mathrm{B} 1 \mathrm{U}$ & 11 & 5.0982 & 0.0000 & 0.0000 & 0.2021 & 0.0051 \\
\hline $\mathrm{B} 1 \mathrm{U}$ & 12 & 5.1520 & 0.0000 & 0.0000 & -0.3857 & 0.0188 \\
\hline $\mathrm{B} 1 \mathrm{U}$ & 13 & 5.2333 & 0.0000 & 0.0000 & -1.3952 & 0.2496 \\
\hline B1U & 14 & 5.6411 & 0.0000 & 0.0000 & 0.3891 & 0.0209 \\
\hline $\mathrm{B} 1 \mathrm{U}$ & 15 & 5.8280 & 0.0000 & 0.0000 & -0.3100 & 0.0137 \\
\hline $\mathrm{B} 2 \mathrm{U}$ & 1 & -0.7983 & 0.0000 & 0.6302 & 0.0000 & 0.0078 \\
\hline $\mathrm{B} 2 \mathrm{U}$ & 2 & 0.4883 & 0.0000 & 0.0681 & 0.0000 & 0.0001 \\
\hline $\mathrm{B} 2 \mathrm{U}$ & 3 & 0.9333 & 0.0000 & -0.4747 & 0.0000 & 0.0052 \\
\hline $\mathrm{B} 2 \mathrm{U}$ & 4 & 1.8534 & 0.0000 & 0.7014 & 0.0000 & 0.0223 \\
\hline $\mathrm{B} 2 \mathrm{U}$ & 5 & 3.5602 & 0.0000 & 1.0649 & 0.0000 & 0.0989 \\
\hline $\mathrm{B} 2 \mathrm{U}$ & 6 & 3.6877 & 0.0000 & 0.1676 & 0.0000 & 0.0025 \\
\hline $\mathrm{B} 2 \mathrm{U}$ & 7 & 4.1799 & 0.0000 & -0.0704 & 0.0000 & 0.0005 \\
\hline $\mathrm{B} 2 \mathrm{U}$ & 8 & 4.3565 & 0.0000 & -0.3293 & 0.0000 & 0.0116 \\
\hline $\mathrm{B} 2 \mathrm{U}$ & 9 & 4.6324 & 0.0000 & -0.1025 & 0.0000 & 0.0012 \\
\hline $\mathrm{B} 2 \mathrm{U}$ & 10 & 5.0162 & 0.0000 & 0.2287 & 0.0000 & 0.0064 \\
\hline $\mathrm{B} 2 \mathrm{U}$ & 11 & 5.1224 & 0.0000 & 0.3864 & 0.0000 & 0.0187 \\
\hline B2U & 12 & 5.5306 & 0.0000 & 0.1450 & 0.0000 & 0.0028 \\
\hline $\mathrm{B} 2 \mathrm{U}$ & 13 & 5.6765 & 0.0000 & 0.5260 & 0.0000 & 0.0385 \\
\hline $\mathrm{B} 2 \mathrm{U}$ & 14 & 5.8154 & 0.0000 & 0.0125 & 0.0000 & 0.0000 \\
\hline B3U & 1 & 3.2691 & -0.0536 & 0.0000 & 0.0000 & 0.0002 \\
\hline B3U & 2 & 3.6288 & -0.0027 & 0.0000 & 0.0000 & 0.0000 \\
\hline
\end{tabular}




$\begin{array}{lcccccc}\text { B3U } & 3 & 4.0333 & -0.0487 & 0.0000 & 0.0000 & 0.0002 \\ \text { B3U } & 4 & 4.2897 & 0.0178 & 0.0000 & 0.0000 & 0.0000 \\ \text { B3U } & 5 & 4.4376 & -0.0121 & 0.0000 & 0.0000 & 0.0000 \\ \text { B3U } & 6 & 4.5776 & -0.0341 & 0.0000 & 0.0000 & 0.0001 \\ \text { B3U } & 7 & 4.6115 & -0.1555 & 0.0000 & 0.0000 & 0.0027 \\ \text { B3U } & 8 & 4.6534 & -0.0711 & 0.0000 & 0.0000 & 0.0006 \\ \text { B3U } & 9 & 4.8221 & 0.0306 & 0.0000 & 0.0000 & 0.0001 \\ \text { B3U } & 10 & 4.8827 & 0.0026 & 0.0000 & 0.0000 & 0.0000\end{array}$

